VOL. 68 (2003) [39-43]

\title{
SUR UN TYPE PARTICULIER DE VALEUR PROPRE DES SOLVARIÉTÉS D'EINSTEIN
}

\author{
Hamid-Reza Fanä̈
}

\begin{abstract}
On montre que la partie nilpotente d'une solvariété d'Einstein standard, dont le type de valeur propre est égal à $\left(1<2 ; d_{1}, d_{2}\right)$, est nécessairement indécomposable si $d_{1}$ et $d_{2}$ sont premiers entre eux.
\end{abstract}

\section{INTRODUCTION}

Nous étudions dans cette note, certaines métriques invariantes à gauche sur les groupes de Lie résolubles.

Nous rappelons que tous les exemples connus de variétés homogènes d'Einstein noncompactes et non-plates sont isométriques aux solvariétés d'Einstein $\left(S, Q_{0}\right)$ où $S$ désigne un groupe de Lie résoluble simplement connexe muni d'une métrique invariante à gauche d'Einstein $Q_{0}$. Essentiellement nous étudions les algèbres de Lie résolubles métriques $(\mathfrak{s}, Q)$ comme nous l'avons fait dans $[3,4]$. Une telle algèbre de Lie métrique est appelée standard $([6])$ si $[\mathfrak{s}, \mathfrak{s}]^{\perp}$ est abélien. On note $\mathfrak{n}:=[\mathfrak{s}, \mathfrak{s}]$ (la partie nilpotente) et $\mathfrak{a}:=\mathfrak{n}^{\perp}$.

Soit $H_{Q} \in \mathfrak{s}$ le vecteur défini par

$$
Q\left(H_{Q}, X\right)=\operatorname{tr} \operatorname{ad}_{X} \quad \text { pour tout } X \in \mathfrak{s .}
$$

Dans la formule de $\operatorname{ric}_{Q}$, l'application $\operatorname{ad}_{H_{Q}}$ apparaît de manière décisive. Nous étudions une propriété de cette application.

Tout d'abord, nous présentons une propriété importante trouvée par J. Heber : soit $(\mathfrak{s},[]$,$) une algèbre de Lie non-unimodulaire résoluble munie d'une métrique d'Einstein$ standard $Q_{0}$. Alors d'après le $[6$, Théorème 4.10$], D_{H_{0}}$ la partie symétrique de ad $H_{0}$ $\epsilon \operatorname{End}(\mathrm{s})$ est aussi une dérivation (et définie positive sur n), où $H_{0}=H_{Q_{0}}$ est non nul (par hypothèse de non-unimodularité).

En d'autre terme, $\left(\mathfrak{s},[],, Q_{0}\right)$ est isométrique à un espace de type Iwasawa. Ceci implique que $\operatorname{ad}_{H_{0}}$ est un opérateur normal de $\left(5, Q_{0}\right)$. La propriété qui nous intéresse est la suivante : pour une telle algèbre de Lie $\left(s,[],, Q_{0}\right)$, il existe un multiple positif unique

Received 22nd October, 2002

Je remercie le Conseil de Recherches de l'Université Technologique Sharif de Téhéran, Iran pour son soutien.

Copyright Clearance Centre, Inc. Serial-fee code: 0004-9727/03 \$A2.00+0.00. 
$H_{1}=\lambda H_{0}$ tel que l'opérateur normal $\left.\operatorname{ad}_{H_{1}}\right|_{\mathrm{n}}$ a des valeurs propres dont les parties réelles $\mu_{1}<\cdots<\mu_{m}$ sont des entiers sans diviseur commun ([6, Théorème 4.14]). Les $\mu_{i}$ sont les valeurs propres de $\left.D_{H_{1}}\right|_{\mathfrak{n}}$. Si $d_{i}, i=1, \ldots, m$ désignent les multiplicités correspondantes, alors on appelle le $2 m$-uplet

$$
\left(\mu_{1}<\cdots<\mu_{m} ; d_{1}, \ldots, d_{m}\right)
$$

le type de valeur propre de solvariété d'Einstein standard associée ([6]).

Dans la partie suivante, nous considérons le cas particulier de type de valeur propre $\left(1<2 ; d_{1}, d_{2}\right)$ où $\mathfrak{n}$ est nécessairement nilpotente de rang deux et $\operatorname{dim} \mathfrak{a}=1$. C'est dans ce cadre que Damek et Ricci ont trouvé leurs exemples harmoniques. Récemment dans [5] une famille continue de solvariétés d'Einstein de courbure sectionnelle négative a été obtenue dans ce cas. Il est intéressant d'étudier les obstructions possibles sur $d_{1}$ et $d_{2}$ lorsqu'il existe une solvariété d'Einstein de type de valeur propre égal à $\left(1<2 ; d_{1}, d_{2}\right)$. Nous montrons que si $d_{1}$ et $d_{2}$ sont premiers entre eux, alors la partie nilpotente d'une telle solvariété est indécomposable.

\section{TyPe DE VAleur PROPRE $\left(1<2 ; d_{1}, d_{2}\right)$}

Soit $S$ un groupe de Lie résoluble connexe et simplement connexe. On note $\mathfrak{s}$ son algèbre de Lie. On désigne par $\operatorname{Der}(\mathfrak{s}) \subset \operatorname{End}(\mathfrak{s})$ l'algèbre de Lie de toutes les dérivations de $\mathbf{s}$.

Supposons que $\mathfrak{s}$ est munie d'un produit scalaire (défini positif) $Q$. Comme nous avons défini auparavant, le vecteur $H_{Q}$ est déterminé par $Q\left(H_{Q}, X\right)=\operatorname{tr} \operatorname{ad}_{X}$ pour tout $X \in \mathfrak{s}$. Il est clair que $H_{Q}$ est perpendiculaire à l'algèbre dérivée $[\mathfrak{s}, \mathfrak{s}]$. Nous rappelons que $S$ (ou s) est appelé unimodulaire si pour tout $X \in \mathfrak{s}, \operatorname{tr} \operatorname{ad}_{X}=0$ ou de manière équivalente si le vecteur $H_{Q}$ s'annule (pour tout $Q$ ).

Le produit scalaire $Q$ induit une métrique riemannienne invariante à gauche sur $S$ que l'on note toujours $Q$. Si le tenseur de courbure de $\operatorname{Ricci} \operatorname{ric}(v, w)=\operatorname{tr} R(\cdot, v) w$ défini à partir de $R$, le tenseur de courbure de Riemann, est un multiple de la métrique $Q$, alors $Q$ est appelée d'Einstein. Si $Q_{0}$ est une métrique d'Einstein sur $(\mathfrak{s},[]$,$) , la constante$ de proportionnalité est non-positive puisque $S$ est non-compact ([2]). De plus, si ric Q $_{Q_{0}}$ s'annule alors $\left(S, Q_{0}\right)$ est une variété plate (voir par exemple [1]). Nous considérons donc le cas

$$
\operatorname{ric}_{Q_{0}}(v, w)=-c \cdot Q_{0}(v, w)
$$

avec $c>0$. La courbure scalaire est alors égale à $s c\left(Q_{0}\right)=-c \cdot \operatorname{dim} S$. Si $[\mathfrak{s , s}]^{\perp}$ (relatif à $Q$ ) est abélien, nous appelons $(\mathfrak{s}, Q)$ une algèbre de Lie standard $([6])$.

Maintenant nous citons la propriété de rationnalité concernant les valeurs propres de $D_{H_{0}}$ mentionnée auparavant dès qu'une métrique d'Einstein standard $Q_{0}$ est fixée. Nous rappelons tout d'abord le lemme crucial suivant (voir le [6, Lemme 4.13]). 
LEMME 2.1. Avec les notations précédentes, si $\left(\mathfrak{s},[],, Q_{0}\right)$ est une algèbre de Lie non-unimodulaire résoluble munie d'une métrique d'Einstein standard $Q_{0}$, alors pour toute dérivation $A \in \operatorname{Der}(\mathbf{s})$, nous avons

$$
c \cdot \operatorname{tr} A=\operatorname{tr}\left(D_{H_{0}} \circ A\right)
$$

REMARQUE. La dérivation symétrique $D_{H_{0}}$ est caractérisée par cette propriété. Notons que le cas $A=D_{H_{0}}$ donne $c=\operatorname{tr} D_{H_{0}}^{2} / \operatorname{tr} \operatorname{ad}_{H_{0}}$.

Le [7, Lemme 1.4(3)] sera également utilisé dans la suite. Rappelons ce lemme.

LEMME 2.2. Soit $(\langle H\rangle \oplus \mathfrak{n}, Q)$ une algèbre de Lie résoluble métrique. $S i \operatorname{ad}_{H_{Q}}$ est symétrique, alors pour tout vecteur $X \in \mathfrak{s}$

$$
\operatorname{ric}_{\mathfrak{s}}(X, X)=\operatorname{ric}_{n}(X, X)-Q\left(\operatorname{ad}_{H_{Q}} X, X\right) .
$$

Maintenant, supposons que $0<\lambda_{1}<\lambda_{2}<\cdots<\lambda_{m}$ sont les valeurs propres distinctes de $\left.D_{H_{0}}\right|_{\mathbf{n}}$ de multiplicités $d_{j}=\operatorname{dim} \mathbf{n}_{j}$ et $\boldsymbol{n}_{j}$ les espaces propres associés dans $\mathfrak{n}$. Nous avons alors :

THÉORÈmE 2.3. (Heber) Il existe une constante positive $\omega$, telle que les valeurs propres de $D_{H_{0}}: \mathfrak{n} \rightarrow \mathfrak{n}$ appartiennent $\mathbf{a} \omega \cdot \mathbb{N}$.

Ceci implique qu'il existe un multiple positif unique $H_{1}=\lambda H_{0}$ tel que l'opérateur $\left.\operatorname{ad}_{H_{1}}\right|_{\mathrm{n}}$ a des valeurs propres dont les parties réelles $\mu_{1}<\cdots<\mu_{m}$ sont des entiers positifs sans diviseur commun. Les $\mu_{i}$ sont les valeurs propres de $\left.D_{H_{1}}\right|_{n}$. Si $d_{i}$ désignent les multiplicités correspondantes, alors on appelle le $2 m$-uplet

$$
\left(\mu_{1}<\cdots<\mu_{m} ; d_{1}, \ldots, d_{m}\right)
$$

le type de valeur propre de solvariété (non-unimodulaire) d'Einstein standard $\left(\boldsymbol{s},[],, Q_{0}\right)$. À l'aide du théorème précédent nous obtenons des obstructions à l'existence d'une telle métrique $Q_{0} \operatorname{sur}(s,[]$,$) avec \operatorname{dim} \mathfrak{a}=1$, dès qu'un type de valeur propre est fixé. En effet, l'espace $a=\langle H\rangle$ étant de dimension 1, nous pouvons parler de type de valeur propre sans fixer une métrique d'Einstein. Dans ce cas les valeurs propres dans un type donné sont celles de $\operatorname{ad}_{H}$ (pour quelques exemples voir [6] 5.3). Nous considérons dans cette partie, le type de valeur propre $\left(1<2 ; d_{1}, d_{2}\right)$ pour $\mathfrak{s}=\langle H\rangle \oplus \mathrm{n}$.

Dans la partie [6, (E) 5.3], il y a déjà un exemple d'une telle $s$ de type en question qui n'admet pas de métrique d'Einstein. Notre résultat fournit une condition d'indécomposabilité lorsque $d_{1}$ et $d_{2}$ sont premiers entre eux. En effet, nous allons montrer que si $(\mathfrak{s},[]$,$) admet une métrique d'Einstein Q_{0}$, alors $\mathfrak{n}=[\mathfrak{s}, \mathfrak{s}]$ est indécomposable. Ceci se fait par la méthode utilisée dans [6]. Rappelons qu'une algèbre de Lie $(n,[]$,$) est$ dite décomposable s'il existe deux sous-algèbres non triviales $\mathfrak{n}_{1}$ et $\mathfrak{n}_{2}$ telles que $\mathfrak{n}=\mathfrak{n}_{1} \oplus \mathfrak{n}_{2}$ et $\left[\mathfrak{n}_{1}, \mathfrak{n}_{2}\right]=\{0\}$. Maintenant nous pouvons montrer le 
THÉORÈme 2.4. Soit $(\mathfrak{s}=\langle H\rangle \oplus \mathfrak{n},[]$,$) une algèbre de Lie résoluble non-$ unimodulaire de type de valeur propre $\left(1<2 ; d_{1}, d_{2}\right)$ où $d_{1}$ et $d_{2}$ sont premiers entre eux. Si s admet une métrique d'Einstein $Q_{0}$, alors $\mathfrak{n}$ est indécomposable.

DÉmonstration: Supposons que $(\mathfrak{s},[]$,$) est munie d'une métrique d'Einstein Q_{0}$. Tout d'abord, nous remarquons que le type de valeur propre est un invariant d'isomérie, i.e. deux solvariétés d'Einstein standards isométriques ont même type de valeur propre. Grâce au [6, Théorème 4.10] que nous avons rapidement expliqué au début de la note, nous sommes sûrs que les deux espaces $\left(\mathfrak{s},[],, Q_{0}\right)$ et $\left(\mathfrak{s},[,]^{+}, Q_{0}\right)$ sont isométriques, où $[,]^{+}=[$,$] sur \mathfrak{n}$ et $\left[H_{0},\right]^{+}=D_{H_{0}}$. Par conséquent, ils ont même type de valeur propre. Ceci nous permet donc de supposer sans perdre de généralité que $\mathrm{ad}_{H_{0}}$ est une dérivation symétrique de $\boldsymbol{s}$.

Nous écrivons maintenant $\mathfrak{n}=\mathfrak{n}_{1} \stackrel{\frac{1}{\oplus}}{\oplus} \mathfrak{n}_{2}$ où $\operatorname{dim} \mathfrak{n}_{i}=d_{i}$ et $\left[\mathfrak{n}, \mathfrak{n}_{2}\right]=\{0\}, 0 \neq\left[\mathfrak{n}_{1}, \mathfrak{n}_{1}\right]$ $\subset \mathfrak{n}_{2}$ ce qui est possible car nous avons

$$
\left.\operatorname{ad}_{H_{0}}\right|_{n_{i}}=i \mu \mathrm{Id}
$$

avec $i=1,2$ pour une constante $\mu$. Notons que ceci entraîne que $(\mathfrak{n},[]$,$) est nilpotente$ de, rang deux. Nous montrons qu'en fait $\left[\mathfrak{n}_{1}, \mathfrak{n}_{1}\right]=\mathfrak{n}_{2}$.

Si ceci n'est pas le cas, alors $n=\left(n_{1} \oplus\left[n_{1}, n_{1}\right]\right) \stackrel{1}{\oplus} n_{0}$ où $0 \neq n_{0} \subset n_{2}$ est abélien. En appliquant le Lemme 2.2 pour $X \in n_{0}$ nous obtenons $c=2 \mu$. D'autre part l'égalité $c=\operatorname{tr} D_{H_{0}}^{2} / \operatorname{tr} \operatorname{ad}_{H_{0}}$ donne

$$
c=\mu \cdot\left(d_{1}+4 d_{2}\right) /\left(d_{1}+2 d_{2}\right)
$$

ce qui contredit l'égalité précédente.

Supposons maintenant que $\mathfrak{n}$ est décomposable. Nous pouvons écrire dans ce cas

$$
\mathbf{n}_{1}=\mathbf{n}^{\prime} \oplus \mathfrak{n}^{\prime \prime}, \mathfrak{n}_{2}=\left[\mathfrak{n}^{\prime}, \mathfrak{n}^{\prime}\right] \oplus\left[\mathfrak{n}^{\prime \prime}, \mathfrak{n}^{\prime \prime}\right]
$$

où $\left[\mathfrak{n}^{\prime}, \mathfrak{n}^{\prime \prime}\right]=\{0\}$ et $\operatorname{dim} \mathfrak{n}^{\prime}=d^{\prime}>0, \operatorname{dim} \mathfrak{n}^{\prime \prime}=d^{\prime \prime}>0\left(d^{\prime}+d^{\prime \prime}=d_{1}\right)$ et $\operatorname{dim}\left[\mathfrak{n}^{\prime}, \mathfrak{n}^{\prime}\right]=\ell^{\prime}$ $>0, \operatorname{dim}\left[\mathfrak{n}^{\prime \prime}, \mathrm{n}^{\prime \prime}\right]=\ell^{\prime \prime} \geqslant 0\left(\ell^{\prime}+\ell^{\prime \prime}=d_{2}\right)$.

Soit $A: \mathfrak{s} \rightarrow \mathfrak{s}$ définie par $A\left(H_{0}\right)=0,\left.A\right|_{n^{\prime}}=\alpha_{1}$ Id, $\left.A\right|_{\mathfrak{n}^{\prime \prime}}=\alpha_{2}$ Id, $\left.A\right|_{\left[\mathfrak{n}^{\prime}, n^{\prime}\right]}=2 \alpha_{1}$ Id et si $\ell^{\prime \prime}>0,\left.A\right|_{\left\{n^{\prime \prime}, n^{\prime \prime}\right]}=2 \alpha_{2}$ Id. Il est clair que $A$ est une dérivation de $\mathbf{s}$. Si $\ell^{\prime \prime}=0$, le Lemme 2.1 nous donne

$$
d^{\prime}(c-\mu) \alpha_{1}+d^{\prime \prime}(c-\mu) \alpha_{2}+2 d_{2}(c-2 \mu) \alpha_{1}=0
$$

et puisque $\alpha_{1}$ et $\alpha_{2}$ sont quelconques, nous obtenons $c-\mu=c-2 \mu=0$ une contradiction. Si $\ell^{\prime \prime}>0$, alors le même argument implique

$$
d^{\prime}(c-\mu) \alpha_{1}+d^{\prime \prime}(c-\mu) \alpha_{2}+2 \ell^{\prime}(c-2 \mu) \alpha_{1}+2 \ell^{\prime \prime}(c-2 \mu) \alpha_{2}=0
$$


et nous obtenons $d^{\prime} / d^{\prime \prime}=\ell^{\prime} / \ell^{\prime \prime}$ ou bien $d^{\prime} /\left(d_{1}-d^{\prime}\right)=\ell^{\prime} /\left(d_{2}-\ell^{\prime}\right)$ et donc $d^{\prime \prime} d_{2}=d_{1} \ell^{\prime}$. Maintenant $d_{2}$ divise $d_{1} \ell^{\prime}$ et puisque $d_{1}$ et $d_{2}$ sont premiers entre eux, $d_{2}$ divise $\ell^{\prime}$, impossible car $d_{2}>\ell^{\prime}$.

REMARque. Il est facile de voir que l'existence d'une métrique d'Einstein $Q_{0}$ impose la condition $d_{2} \leqslant d_{1}\left(d_{1}-1\right) / 2$. De même si $d_{1}$ est impaire, alors $d_{2}$ ne peut pas être égal à 1 ou 2 ni égal à $d_{1}\left(d_{1}-1\right) / 2-1$ ou $d_{1}\left(d_{1}-1\right) / 2-2([5])$. D'autres obstructions ne sont pas connues à ce sujet. Par contre, pour les cas possibles, il y a des exemples, comme le cas $d_{2}=2 r$ et $d_{1}=k d_{2}$, pour tous $r, k$.

\section{REFERENCES}

[1] L. Bérard Bergery, 'Sur la courbure des métriques riemanniennes invariantes des groupes de Lie et des espaces homogènes', Ann. Sci. École. Norm. Sup. 11 (1978), 543-576.

[2] A.L. Besse, Einstein manifolds (Springer-Verlag, Berlin, 1987).

[3] H.-R. Fanaï, 'Espaces homogènes d'Einstein non-compacts', Geom. Dedicata 80 (2000), $187-200$.

[4] H.-R. Fanaï, 'Variétés homogènes d'Einstein de courbure scalaire négative : Construction à l'aide de certains modules de Clifford', Geom. Dedicata 93 (2002), 77-87.

[5] C.S. Gordon et M.M. Kerr, 'New homogeneous Einstein metrics of negative Ricci curvature', Ann. Global Anal. Geom. 19 (2001), 75-101.

[6] J. Heber, 'Noncompact homogeneous Einstein spaces', Invent. Math. 133 (1998), 279-352.

[7] T. Wolter, 'Einstein metrics on solvable groups', Math. Z. 206 (1991), 457-471.

Department of Mathematical Sciences

Sharif University of Technology

P.O.Box 11365-9415

Tehran

Iran

e-mail: fanai@sharif.ac.ir 